\title{
Die Germanistik im mehrsprachigen Bildungssystem Belgiens
}

Achim Küpper und Torsten Leuschner

\section{Einleitung}

Die belgische Hochschullandschaft ist mindestens ebenso kompliziert wie das notorisch komplexe belgische Staatsgebilde. Neben den ideologischen Gegensatz zwischen den katholischen und den nichtkonfessionellen Interessengruppen treten die unterschiedlichen Belange der Sprachgemeinschaften des Landes. Aufgrund dieser Konstellation hat sich in Belgien in einem schwierigen Reformprozess ein mehrschichtig-zentrifugales Föderalismusmodell herausgebildet, ${ }^{1}$ dessen Ausgestaltung sich im Hochschulwesen zudem mit der Bologna-Reform und weiteren Faktoren überschneidet. Die Fallstudie, die sich daraus ergibt, wird noch interessanter, wenn man sie aus Sicht eines sprachlich-kulturell bestimmten Studienfaches wie der Germanistik betrachtet.

Der heutige trilinguale Föderalstaat Belgien untergliedert sich in insgesamt drei Sprachgemeinschaften mit je eigener Kompetenz für das Bildungswesen: die flämische (d.h. niederländischsprachige), die französischsprachige und die deutschsprachige Gemeinschaft. Die ersten beiden können eine verhältnismäßig hohe Anzahl germanistischer Institute an Universitäten vorweisen. Die deutschsprachige Gemeinschaft als die mit Abstand kleinste der drei verfügt dagegen über keine eigene Universität. Auf universitärer Ebene wird Germanistik in Belgien daher durchweg als Fremdsprachenfach organisiert. Allerdings ist an frankophonen Universitäten in der Nähe zum deutschsprachigen Gebiet ein Anteil muttersprachlicher Studierender von bis zu 30\% zu verzeichnen. Das führt unter anderem zu sprachlich gemischten TeilnehmerInnengruppen in den Kursen.

\section{Belgien: vom Sprachenstreit zum Bildungsföderalismus}

Das Königreich Belgien geht im Wesentlichen auf den südlichen Teil (ohne das Großherzogtum Luxemburg) der historischen Niederlande zurück, der sich 1830 vom kurzlebigen ,Vereinigten Königreich der Niederlande' abspaltete, um unter Leopold I. von Sachsen-Coburg ein selbständiges Königreich zu bilden. ${ }^{2}$ Die Sprache der höheren Bildung war danach in ganz Belgien bis in die erste Hälfte des 20. Jh.s hinein das Französische. Auf politischer Ebene wurde die Trennung zwischen dem niederländischsprachigen, dem französischsprachigen und dem deutschsprachigen Bildungswesen (jeweils mit einem eigenen Kultusministerium) sogar erst 1983 im Rahmen der Föderalisierung Belgiens besiegelt. Dieser Föderalisierungsprozess brachte die charakteristische Zweigliedrigkeit des

\footnotetext{
${ }^{1}$ Siehe u.a. Woyke 2009; Rochtus 2010. Über Belgien und seine Geschichte informieren u.a. Kroll 2007; Erben 2009; Witte [u.a.] 2016 (auch in französischer und englischer Übersetzung). Aktuelle und Hintergrundinformationen finden sich auf http://www.belgieninfo.net sowie auf http://www.flanderninfo.be [letzter Aufruf: 22.12.2016], dem deutschsprachigen Nachrichtenportal des flämischen öffentlich-rechtlichen Rundfunks VRT.

${ }^{2}$ Den belgischen Sprachenstreit behandeln Krämer 2010; Witte/Van Velthoven 2010; Lamarcq/Rogge 1998. Letztere beide liegen auch in französischer Übersetzung vor.
} 
belgischen Föderalismus (vgl. Abb. 1) hervor, die zwischen Sprachgemeinschaften (der flämischen, französischsprachigen und deutschsprachigen) und Regionen (Flandern, Wallonien und Brüssel) unterscheidet. AbiturientInnen aus der Deutschsprachigen Gemeinschaft (DG), deren gut 70.000 Einwohner im äußersten Osten Belgiens zwischen Aachen und Luxemburg wohnen, ${ }^{3}$ studieren entweder an einer französisch- oder niederländischsprachigen Universität oder in Deutschland.

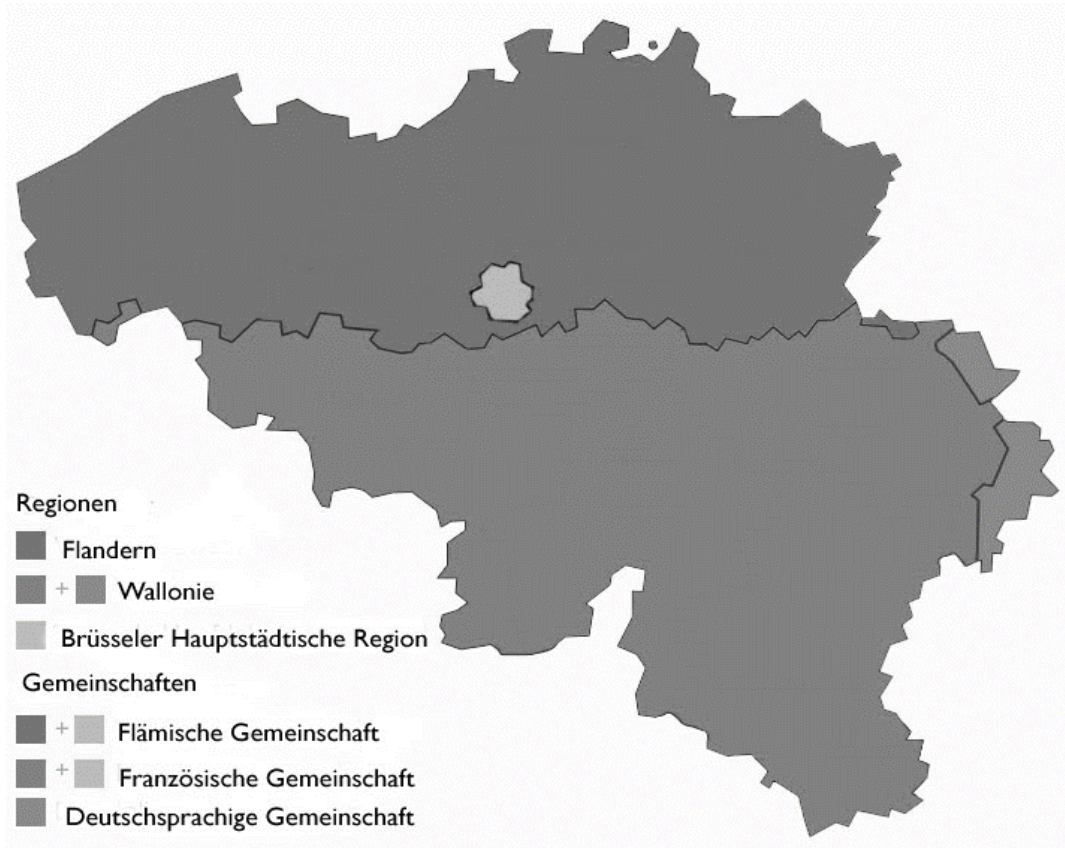

Abb. 1: Die belgischen Regionen und Gemeinschaften

\section{Studienorte}

Derzeit gibt es in Belgien 11 Universitäten mit Germanistik. Sowohl BA- als auch MA-Studiengänge werden an der Universiteit Gent (UGent), der Universiteit Antwerpen (UA), der Vrije Universiteit Brussel (VUB), der Université Libre de Bruxelles (ULB), der Université Catholique de Louvain (UCL), der Katholieke Universiteit Leuven (KUL) und der Université de Liège (ULg) angeboten; an der Université de Namur (UNamur) und der Université Saint-Louis - Bruxelles (USL) gibt es in der Germanistik und den anderen Geisteswissenschaften nur BA-Studiengänge. An der Université de Mons (UMons) gibt es nur einen Übersetzer- und Dolmetscherstudiengang mit Deutsch, an der Universiteit Hasselt gibt es gar keine geisteswissenschaftlichen Studiengänge.

Unterschiede zwischen den Universitäten der beiden großen Sprachgemeinschaften gibt es in Germanistik weiterhin bei den Studierendenzahlen: Sie liegen in Flandern zwischen etwa 150 (UGent) und etwa 40 (VUB), in Wallonien zwischen etwa 90 (Lüttich) und einem runden Dutzend (USL). Die Differenz hängt zumindest teilweise mit der Tatsache zusammen, dass im frankophonen

\footnotetext{
${ }^{3}$ Über die DG informieren z.B. Förster [u.a.] 2003; Schwieren-Höger/Sackermann 2008; Wenselaers 2008; Berge/Grasse 2003. Ferner die Tageszeitung Grenz-Echo (http://www.grenzecho.net [letzter Aufruf: 22.12.2016]).
} 
Sekundarschulwesen weniger SchülerInnen mit der deutschen Sprache in Kontakt kommen: Während in der französischen Gemeinschaft in den letzten Jahren ca. 3\% der SchülerInnen Deutsch lernen (als erste, zweite oder dritte Fremdsprache) ${ }^{4}$ sind es in Flandern ca. 17\% - durchweg als dritte Fremdsprache nach Französisch und Englisch und in zunehmender Konkurrenz mit Spanisch. 17\% sind auch im internationalen Vergleich viel, dennoch ist auch dieser Wert das Ergebnis eines langfristigen Rückgangs. Im Rahmen des Regionalförderplans (des sog. Marshallplans) für Wallonien werden seit einigen Jahren Maßnahmen zur Förderung der Kenntnisse anderer Landessprachen ergriffen, u.a. mittels Sprachaufenthalten in Flandern, den Niederlanden oder Deutschland. ${ }^{5}$ Im frankophonen Sekundarschulwesen wird seit einigen Jahren auch der sogenannte Immersionsunterricht stark gefördert, im flämischen Schulwesen gibt es neuerdings ähnliche, wenn auch zurückhaltendere Tendenzen.

Bemerkenswerte innerbelgische Unterschiede betreffen schließlich noch das Verhältnis der sprach- und literaturwissenschaftlichen Studiengänge zur Übersetzerausbildung, die ebenfalls an den meisten Universitäten angeboten wird. Dabei haben sich die Ausbildungsgänge für ÜbersetzerInnen und DolmetscherInnen an den französischsprachigen Universitäten im Allgemeinen eng an die Sprach- und Literaturwissenschaft angeschlossen, während ihre Selbstständigkeit in Flandern - teils im Rahmen breiterer Studiengänge der Angewandten Linguistik o. Ä. - vorerst unangetastet geblieben ist.

Inhaltlich zeichnet sich an den meisten Germanistikabteilungen eine stärkere Orientierung auf einen vielfältiger gewordenen Arbeitsmarkt ab. Das Lehramt ist längst nicht mehr die einzige Berufswahl der meisten GermanistInnen; viele landen in der Privatwirtschaft, im Kultursektor oder bei den Medien. Im Hinblick darauf werden an vielen Universitäten verstärkt anwendungsorientierte Aufbaustudiengänge wie Geschäftskommunikation und Journalismus angeboten. Auch im Germanistikstudium spielen praktische Sprachkenntnisse eine stärkere Rolle als zuvor, allerdings ohne dass deshalb die Landeskunde im Pensum an Bedeutung zunähme.

\section{Globaler Rückgang und hohe Nachfrage: Paradoxien des Deutschen in Belgien}

Schwierigkeiten bereitet der globale Rückgang des Deutschen, der v.a. an den frankophonen Universitäten jüngst unter anderem durch eine stärkere ,Praxisorientierung' der Studieninhalte abgefangen werden soll. Dabei wird der Akzent von traditionell geisteswissenschaftlichen zu eher angewandten Bereichen hin verlagert, etwa in der Veranstaltung von Übersetzungslehrgängen, was zum Teil in Zusammenarbeit zwischen Universität und Fachhochschule geschieht. An den flämischen

\footnotetext{
${ }^{4}$ Vgl. http://www.enseignement.be/index.php?page=23793\&navi=56\&rank_page=23793 [letzter Aufruf: 22.12.2016].

${ }^{5}$ Vgl. u.a. http://www.efbelgique.be/top/plan-marshall/ sowie https://www.leforem.be/particuliers/stages-immersionlangues.html [letzter Aufruf: 22.12.2016].
} 
Universitäten wird dagegen weiterhin auf die Unabhängigkeit der Übersetzerstudiengänge, die erst vor einigen Jahren von den Fachhochschulen an die Universitäten verlagert wurden, geachtet und neigen die geisteswissenschaftlichen Studiengänge dazu, sich einer stärkeren Praxisorientierung eher zu widersetzen. Diese insgesamt zunehmende Praxisorientierung und ihre Begleiterscheinungen im Hochschulwesen sind Teil einer weit globaleren Tendenz in Lehre und Forschung.

Dabei ist die Nachfrage nach Deutschkenntnissen in Belgien weiterhin sehr hoch. Das liegt u.a. an der geographischen Nähe und engen wirtschaftlichen Verbindung zu Deutschland. Deutschland ist nach wie vor der wichtigste Handelspartner Belgiens; viele Betriebe am Arbeitsstandort Belgien suchen Arbeitskräfte mit Deutschkenntnissen. Hierin liegt ein überaus deutliches Argument für das Erlernen und das Studium des Deutschen. Dagegen wollen aber nur mäßig viele Menschen Deutsch lernen, wobei die Zahl in den verschiedenen Landesteilen stark variiert. Nicht allein in der an Deutschland angrenzenden Provinz Lüttich, sondern auch in anderen Teilen Belgiens ist der Bedarf an Arbeitskräften mit Deutschkenntnissen so hoch und das BewerberInnenangebot so niedrig, dass manche Betriebe keine passenden KandidatInnen finden.

Dieses Missverhältnis erklärt sich zum einen dadurch, dass das Deutsche mit einigen Schwierigkeiten in seiner Wahrnehmung zu kämpfen hat: mit der angeblichen Schwierigkeit der Sprache, mit einem allgemeinen Image, das beim Deutschen negativer ausfällt als bei manchen anderen Sprachen, oder auch mit dem Umstand, dass Deutsch in dem an sich schon polyglotten Land Belgien erst als zweite oder oft dritte Fremdsprache erlernt wird. Zum anderen ist das Argument der hohen Nachfrage nicht so ausschlaggebend und auch nicht so bekannt, wie es eigentlich sein sollte.

\section{Ausblick}

Wie sich die Studierendenzahlen in der Germanistik auch entwickeln werden, eines steht fest: Überall in Belgien ist der Bedarf an Deutsch in der Wirtschaft hoch. Nicht selten bleiben Arbeitsplätze unbesetzt oder müssen Betriebe eigene Fortbildungsmaßnahmen finanzieren, weil es an BewerberInnen mit ausreichenden Deutschkenntnissen fehlt; die Universitäten und Hochschulen passen sich an und richten neue Studiengänge wie etwa Wirtschaftskommunikation ein (s.o.). In der Französischsprachigen Gemeinschaft ist schon jetzt eine stärkere Berufs- und Arbeitsmarktorientierung in der Studienwahl feststellbar: Die StudienanfängerInnen entscheiden sich nun z.B. öfter für Deutsch im Rahmen einer Dolmetscher- und Übersetzerausbildung als für ein Germanistikstudium, und auch die neuen wirtschaftsorientierten MA-Studiengänge kommen bei ihnen gut an. Die zunehmende Betonung der Mehrsprachigkeit der AbsolventInnen vor allem im frankophonen Hochschulwesen könnte für die deutsche Sprache ebenfalls förderlich sein, wenn 
Germanistik z.B. als dritte Sprache (Wahlfach oder mineure) gewählt werden kann. In Flandern ist diese Möglichkeit bisher nur in begrenztem Maße gegeben.

Dabei lassen sich gerade in Belgien einige der scheinbaren Nachteile der Ausgangssituation in Vorteile umkehren: Die geografische Kleinheit des Landes (bei immerhin fast 11 Millionen Einwohnern) kommt einem engeren, persönlicheren Kontakt und einer besseren Vernetzung zugute; die sprachlich-politische Zerklüftung und Zerrissenheit des Landes führt bei allen Schwierigkeiten auch zu einer Landschaft des Pluralismus jenseits der Uniformität. Belgien ist ein bescheidenes Land im Zentrum Europas. Mit seiner Hauptstadt Brüssel befindet es sich aber nicht allein im topografischen, sondern ebenso im politischen Mittelpunkt der EU. Als mehrsprachiger und multikultureller Schmelztiegel bildet es eine Art Europa im Kleinen. Wo dieses Land seine Vorteile nutzt, wird es zu einem ebenso vielversprechenden wie vielsprachigen Begegnungsraum und zu einem Ort der Vielfalt. Für das Fach Deutsch liegt hier ein großes Potenzial, das es in Zukunft noch stärker auszuschöpfen gilt.

Zur Nutzung eines solchen Potenzials versucht nicht zuletzt auch der Belgische Germanistenund Deutschlehrerverband (BGDV) einen Beitrag zu leisten: Er versammelt Mitglieder aller Sprachgemeinschaften sowohl aus dem Sekundar- als auch aus dem Hochschulwesen, und arbeitet am wissenschaftlichen und didaktischen Austausch sowie an der interpersonalen Kommunikation von GermanistInnen und allen an der deutschen Sprache oder Kultur Interessierten in einem paradigmatisch mehrsprachigen und plurikulturellen Land. Für dieses Engagement steht unter anderem auch die jüngste Neuakzentuierung der vom BGDV seit 1975 herausgegebenen Fachzeitschrift Germanistische Mitteilungen (Heidelberg: Universitätsverlag Winter, 2 Hefte pro Jahr), die sich in Zukunft verstärkt der fachlichen und wissenschaftlichen Auseinandersetzung mit Aspekten der deutschen Sprache, Literatur und Kultur in mehrsprachigen und transnationalen Kontexten verschreiben wird. Damit wollen die Germanistischen Mitteilungen zur Erforschung und Diskussion eines Themenkomplexes beitragen, der im Zentrum eines plurikulturellen Zusammenhangs in der globalisierten, digitalisierten und medialisierten Welt steht und der in Europa heute mehr denn je des dialogischen Austauschs, der wissenschaftlichen Vernetzung und der fachlichen Kommunikation bedarf.

\section{Literatur}

Berge, Frank/Grasse, Alexander: Belgien - Zerfall oder föderales Zukunftsmodell? Der flämischwallonische Konflikt und die Deutschsprachige Gemeinschaft. Opladen 2003.

Erben, Michael: Die Deutschen und ihre Nachbarn. Belgien und Luxemburg. München 2009. 
Förster, Stephan [u.a.]: Die deutschsprachige Gemeinschaft Belgiens - das kleinste Bundesland in der Europäischen Union. In: Jahrbuch des Föderalismus (2003), S. 207-218.

Krämer, Philipp: Der innere Konflikt in Belgien. Sprache und Politik. Geschichte und Gegenwart der mehrsprachigen Gesellschaft. Saarbrücken 2010.

Kroll, Johannes: Belgien. Geschichte - Politik - Kultur. Münster 2007.

Lamarcq, Danny/Rogge, Marc (Hgg.): De taalgrens - van de oude tot de nieuwe Belgen. Leuven 1998. Rochtus, Dirk: Belgien - Föderation mit Sollbruchstellen. In: Regional Governance in EU-Staaten. Hg. von Roland Sturm/Jürgen Dieringer. Opladen 2010, S. 63-78.

Schwieren-Höger, Ulrike/Sackermann, Jörn: Ostbelgien und die Deutschsprachige Gemeinschaft Belgiens. Bilder - Spuren - Hintergründe. Eupen 2008.

Wenselaers, Selm: De laatste Belgen. Een geschiedenis van de Oostkantons. Amsterdam 2008.

Witte, Els [u.a.]: Politieke geschiedenis van België van 1830 tot heden. Antwerpen 2016.

Witte, Els/Van Velthoven, Harry: Strijden om taal. De Belgische taalkwestie in historisch perspectief. Kapellen 2010.

Woyke, Wichard: Das politische System Belgiens. In: Die politischen Systeme Westeuropas. Hg. von Wolfgang Ismayr. Wiesbaden 2009, S. 451-482.

Dr. Achim Küpper, Université du Luxembourg, achim.kuepper@uni.lu; Prof. Dr. Torsten Leuschner, Universiteit Gent, torsten.leuschner@ugent.be 